\title{
Prevalence and Factors Associated With Herbal Medicine Use among HIV Positive Patients on Highly Active Antiretroviral Therapy in Selected Hospitals in Nairobi County
}

\author{
Aaron Mungiria ${ }^{1 *}$ Simon Karanja ${ }^{2}$ Lawrence Muthami $^{3}$ Charles Mutai ${ }^{4}$ \\ 1. Institute of Tropical Medicine and Infectious Diseases (ITROMID) \\ 2. College of Health Sciences, Jomo Kenyatta University of Agriculture and Technology \\ 3. Centre for Public Health Research, Kenya Medical Research Institute \\ 4. Centre for Traditional Medicine and Drug Research, Kenya Medical Research Institute \\ * E-mail of the corresponding author: aaronmurithi@yahoo.com
}

\begin{abstract}
Background: Traditional remedies have been used for many years in Africa to treat various ailments before the introduction of conventional medicines into the continent. Concurrent use of traditional herbal medicines (THM) with highly active antiretroviral therapy (HAART) is widespread among HIV infected patients. Studies done in different parts of the world show conflicting health outcomes among HIV positive patients using HAART and herbal medicine concurrently with some reporting better health outcomes while others report increased risk of adverse drug reactions. However, the extent of THM use is not known in most settings in Sub-Saharan Africa Kenya included. This study aimed to determine the prevalence and factors associated with THM use among HIV infected patients on highly active antiretroviral therapy (HAART) attending two comprehensive care centers in Nairobi County.
\end{abstract}

Methods: A descriptive cross-sectional study was conducted at Kenyatta National Teaching and Referral Hospital and Mbagathi District Hospital. From the two centers a total of 370 participants were selected via systematic random sampling. The participants included were those aged 18 years and above, on HAART, and had consented to participate in the study. Data was collected using an interviewer-administered semi-structured questionnaire seeking information on herbal medicine use, socio-demographic and economic factors associated with herbal medicine use and the association between herbal medicine use and occurrence of adverse drug reactions. THM use referred to someone who had ever used or was currently using herbal medicine while on highly active antiretroviral therapy (HAART) by the time of the study. Data was captured in excel sheet and exported to STATA for analysis.

Results: The study established that the prevalence of herbal medicine use among HIV positive patients taking HAART was $15.5 \%$. About $77.8 \%$ of the patients who had used herbal medicine had never disclosed to the doctor or any health care worker at the CCC about their herbal medicine use. Herbal medicine use led to decreased adherence to HAART. The longer the duration between HIV diagnosis and HAART start, the more likely a HIV positive patient was to use herbal medicine. Patients who use herbal medicine with HAART were more likely to experience adverse drug reactions. There were $59.7 \%$ participants who had good /fair adherence while $40.3 \%$ had poor adherence. Majority of those who had poor adherence had significantly higher proportion of side effects as compared to those had good/fair adherence.

Conclusion: The prevalence of THM use among participants on HAART was high. This raises clinical and pharmacological concerns that need attention by the health care service providers.

Keywords: Herbal Medicine, HIV, Antiretroviral Therapy

DOI: $10.7176 / \mathrm{JBAH} / 9-4-11$

\section{Introduction}

Traditional remedies have been used for many years in Africa to treat various ailments before the introduction of conventional medicines into the continent. The term "traditional medicine" refers to ways of protecting and restoring health that existed before the arrival of modern medicine. Thus, in all countries of the world there exists traditional knowledge related to the health of humans and animals. The importance of traditional medicine (TM) as a source of primary healthcare was first officially recognized by the World Health Organization (WHO) in the Primary Health Care Declaration of Alma Ata (1978) and has been globally addressed since 1976 by Traditional Medicine Program of the WHO.

The wide spread use of traditional medicine (TM) among both rural and urban population could be attributed to 
cultural acceptability, physical accessibility and economic affordability, as well as efficacy against certain types of diseases, as compared to modern medicine (Mukherjee, 2002).

In Kenya, patients would opt for traditional herbal medicine because the traditional herbal medicine practitioners are more accessible than the trained doctors. A large number of the rural poor, in particular, turn to traditional medical practitioners for certain conditions, when access to allopathic health workers is restricted (Lambert et al., 2011)

In Africa many HIV patients use traditional herbal medicine for primary health care or management of side effects (Babb et al, 2007). Traditional beliefs, inconsistent access or unavailability of antiretroviral drugs has led to increase in the use of herbal remedies among HIV-infected individuals in Africa in the past decade (Maponga et al., 2007). There is no ultimate cure for HIV and this has led to people trying different things in an attempt to get cured. Some of the herbal drugs have been reported to cause adverse effects and there have been reports of drug interactions with antiretroviral drugs (Izzo, 2004). These herbal substances could induce enzymes involved in the metabolism of antiretroviral drugs resulting in low plasma concentrations of the drugs or alternatively inhibit the enzymes resulting to higher plasma concentrations of the drugs which would exacerbate their toxicity (Lee et al., 2006).

These life prolonging HAART have several significant side effects and the management of these side effects remains a major challenge especially in the developing countries and this has also contributed to the use of complementary and alternative medicines (UNAIDS, 2008). Herbal medicines do present various health challenges. The multiplicity of these medicines leads not only to potentially severe side effects, but also to clinically significant interactions between ARVs and other medicines. In Canada, majority of the patients who reported HAART related side effects especially neuropathy, also used complementary or alternative medicine (Agnoletto et al., 2006). Use of HAART concurrently with traditional herbal medicine may lead to drug interactions undermining the effectiveness of antiretroviral drugs (Lee et al., 2006).

HIV patients on HAART may not inform medical practitioners about their herbal medicine use as they believe it is not important and taking herbal medicine together with prescribed drugs is harmless especially if for different problems or fear of being treated differently by the healthcare providers (Vickers et al., 2006). Even though there is a general feeling that herbal medicines are safe, a review of toxicity and quality control issues of herbal medicines in Kenya reveal the existence of contamination of herbal medicines with heavy metals, undocumented pharmaceuticals, misidentified plants, pesticides, excessive levels of microorganisms or their toxins and other noxious organic materials as well as high bacteria content that poses serious health risks (Maina et al., 2013).

The standard anti-retroviral drugs (ARVs) used for the treatment of HIV/AIDS have significant side effects resulting in lack of adherence and the emergence of multidrug resistant viral strains. The side effects experienced by HIV patients lead to distrust of conventional therapy and are likely to turn to complementary and alternative medicine (Hsiao et al., 2003). Negative attitude towards the effectiveness of HAART and greater desire for medical information by HIV infected patients makes them more likely to use complementary and alternative medicines which have the potential to cause adverse effects (Lee et al., 2006).

This study was therefore conducted to determine prevalence and factors associated with herbal medicine use among HIV positive patients on highly active antiretroviral therapy in selected hospitals in Nairobi county

\section{Methodology}

\subsection{Study design}

The research adopted a facility based descriptive cross-sectional study which recruited HIV positive patients on HAART aged 18 years and above attending Kenyatta National Teaching and Referral Hospital CCC and Mbagathi District Hospital CCC between June - July 2015. A semi-structured questionnaire was administered to patients who met the selection criteria. Participants received information on the study including the time required for questionnaire completion and assured anonymity of their responses.

\subsection{Study area}

The study was conducted at Kenyatta National Hospital(KNH) and Mbagathi District Hospital both in Nairobi County. Mbagathi District Hospital is located approximately 4 kilometers from the city center. It has a bed capacity 
of 200 and offers a variety of services including Antiretroviral Therapy, Curative In-patient Services, Family Planning, HIV Counseling and Testing, Immunization and many others. The facility is easily accessible from the city center and is well known as it was the district referral center giving it a wide catchment area. It has a history of being an infectious disease hospital hence it popularity among Nairobi residents. It serves close to 4000 HIV positive patients taking HAART making it an ideal center for the study.

$\mathrm{KNH}$ is the largest public health facility and is located in the city about 3 kilometers from the city center. It is about 112 years old. It covers an area of 45.7 hectares and within the KNH complex are College of Health Sciences (University of Nairobi); the Kenya Medical Training College; Kenya Medical Research Institute (KEMRI) and National Laboratory Service .It has 50 wards, 22 out-patient clinics, 24 theatres and Accident \& Emergency Department. It has a total bed capacity of 1800 out of which 209 beds are for the Private Wing. It is accessible to the majority of the population in the county due to its strategic geographical location and the health capacity of the facility. It is the national referral hospital and all the complicated medical conditions that cannot be managed in other hospitals are usually referred to KNH. The facility serves over $5000 \mathrm{HIV}$ positive patients taking HAART.

\subsection{Subject selection}

The study participants from the two sites were selected by Systematic random sampling. We recruited 160 participants from Mbagathi and 210 from $\mathrm{KNH}$ weighted to the number of patients that usually received treatment at each facility. The subjects recruited into the study were those that were HIV positive, above 18 years, taking HAART from the study sites, able to respond to study questions, willing to participate in the study and were Nairobi residents.

\subsection{Data collection}

Primary data was obtained using an interviewer administered semi structured questionnaire. The questionnaire was prepared in both English and Swahili languages. The questionnaire was administered to the participants and the responses were written down and it approximately took 20 minutes to complete the questionnaire.

The type of data collected included demographic characteristics of the participants; age, sex, religion, highest level of education attained, marital status, drug related factors including adherence to HAART, duration on HAART, and side effects; individual factors including familiarity with herbs, beliefs in the usefulness of THM, why they started using THM, what herb was taken and how. Medication adherence was assessed using the validated Morisky medication adherence scale in outpatient setting (Morisky et al., 2008).

\subsection{Ethical issues}

The study was approved for research by KEMRI Scientific Steering and Ethical Review Committees, KNH ethics review committee and the medical superintendent Mbagathi District Hospital.

\subsection{Data management and analysis}

All study participants received a unique participant identification number that was recorded on the questionnaire. Collected data from the study was checked for accuracy and completeness. The data was stored in flash disks, compact disks and a laptop for back up before and after analysis. Data on the questionnaire was kept under lock and key while electronically stored data was password protected. The data was keyed in Microsoft Excel and then exported to STATA for analysis.

Dependent variables include prevalence of herbal medicine use and occurrence of ADRs. Chi square test was computed for categorical variables to test for association between predictor and outcome.

All variables observed to be significant were subjected to multivariate regression analysis to test for interaction. Level of significance was determined at $5 \%$ (p-value $<0.05$ )

Software used was SPSS. 


\section{Results}

\subsection{Socio-demographic and economic characteristics of patients on HAART}

A total of 370 participants were recruited into the study. There were 136(36.8\%) males and 234(63.2\%) Females. A total of $8(2.2 \%)$ participants were below 25 years, 41 (11.3\%) were $25-29$ years, $52(14.4 \%)$ were $30-34$ years, $48(13.4 \%)$ were $35-39$ years, $72(19.9 \%$ )were $40-44$ years, $69(19 \%)$ were $45-49$ years, $41(11.3 \%)$ were $50-54$ years, and 31(8.6\%) participants were aged over 55years. The married participants were 208(61.5\%), single 78 $(23 \%)$, divorced $18(5.3 \%)$ and $34(10 \%)$ participants were separated. A total of $125(33.9 \%)$ participants had acquired tertiary education, $163(44.2 \%)$ had secondary school, $70(19 \%)$ had primary and $11(3 \%)$ had no education. With regard to employment status $113(32 \%)$ participants were in private business, $170(48 \%)$ were employed and $70(19.8 \%)$ were unemployed. There were $7(2.4 \%)$ participants who had a monthly household income less than Ksh5000, 29(10\%) earned between ksh5000-ksh9999, 71(24.48\%) earned ksh10000-19999, 70(24.1\%) earned ksh20000-29999, 28(9.7\%) earned ksh30000-39999, 20(6.9\%) earned 40000-49999 and 65(22.4\%) had a monthly income above ksh50000. (Table 1).

Table 1: Socio-demographic and economic characteristics of patients on HAART

\begin{tabular}{llll}
\hline Variable & & Frequency & percentage \\
\hline Gender & Male & 136 & 36.76 \\
Age Category in Years & Female & 234 & 63.24 \\
& Below 25 & 8 & 2.21 \\
& $25-29$ & 41 & 11.33 \\
& $30-34$ & 52 & 14.36 \\
& $35-39$ & 48 & 13.26 \\
& $40-44$ & 72 & 19.89 \\
Marital Status & $45-49$ & 69 & 19.06 \\
& $50-54$ & 41 & 11.33 \\
Level of education & 55 and above & 31 & 8.56 \\
& Single & 78 & 23.08 \\
& Married & 208 & 61.54 \\
& Divorced or Separated & 52 & 15.39 \\
Employment status & No education & 11 & 2.98 \\
& Primary school & 70 & 18.97 \\
& Secondary school & 163 & 44.17 \\
Monthly household income (Ksh) & Tertiary education & 125 & 33.88 \\
& Unemployed & 70 & 19.83 \\
& Employed & 170 & 48.16 \\
& Private business & 113 & 32.01 \\
& Below 9999 & 36 & 12.41 \\
& $10000-19999$ & 71 & 24.48 \\
& 20000-29999 & 70 & 24.14 \\
& $30000-39999$ & 28 & 9.66 \\
& $40000-49999$ & 20 & 6.90 \\
& Above 50000 & 65 & 22.41 \\
\hline
\end{tabular}

\subsection{Adherence, side effects and HAART start characteristics of patients on HAART}

There were 54(14.8\%) participants who were started HAART within 3 years of HIV diagnosis, 71(19.4\%) were started HAART between 3-5 years, $110(30 \%)$ between 6-8 years, and 131(35.8\%) were started HAART more than 9 years after HIV diagnosis. There were 221(59.7\%) participants who achieved good /fair adherence while $149(40.3 \%)$ had poor adherence. After starting HAART, 228(62\%) participants reported to have experienced side effects while 140(38\%) didn't experience any side effects. (Table 2). 


\section{Table 2: Adherence, side effects and HAART start characteristics of patients on HAART}

\begin{tabular}{llll}
\hline Variable & & Frequency & Percentage \\
\hline $\begin{array}{l}\text { Duration in years between HIV diagnosis } \\
\text { and HAART start }\end{array}$ & Less than 3 years & 54 & 14.75 \\
& & & \\
& $3-5$ & 71 & 19.40 \\
& $6-8$ & 110 & 30.05 \\
Adherence & More than 9 years & 131 & 35.79 \\
& Good/ Fair & 221 & 59.73 \\
Side effects after HAART start & Poor & 149 & 40.27 \\
& Experienced side effects & 228 & 61.96 \\
& Didn't experience side effects & 140 & 38.04 \\
\hline
\end{tabular}

\subsection{Prevalence of herbal medicine use among HIV positive patients on HAART}

The Prevalence of herbal medicine use among HIV positive patients on highly active antiretroviral therapy was $15.5 \%$. (Table 3 ).

Table 3: Prevalence of herbal medicine use among HIV positive patients on HAART

\begin{tabular}{lll}
\hline Herbal \& HAART use & Frequency & Percent \\
\hline No & $\mathbf{3 0 5}$ & $\mathbf{8 4 . 4 9}$ \\
Yes & $\mathbf{5 6}$ & $\mathbf{1 5 . 5 1}$ \\
\hline
\end{tabular}

3.4 Socio-demographic, economic factors and herbal medicine use among HIV positive patients taking HAART.

The study established that $17 \%$ of HIV positive women and $12.9 \%$ of HIV positive men taking HAART used herbal medicine. It was observed that $12.1 \%$ HIV positive patients aged 18-35 years taking HAART used herbal medicine while $16.8 \%$ of those aged above 35 years used herbal medicine. (Table 4 ).

\subsection{Adherence, side effects, HAART start and herbal medicine use among HIV positive patients taking HAART.}

The longer the duration between HIV diagnosis and HAART start, the more likely a HIV positive patient was to use herbal medicine $(\mathrm{P}<0.05)$. Patients who were started HAART more than 9 years after HIV diagnosis had higher odds of using herbal medicine (OR: 4.6, 95\% CI: 1.283-12.045). Patients who had poor adherence are more likely to use herbal medicine. $(\mathrm{P}<0.01)$. The use of herbal medicine with HAART increases the risk of side effects $(\mathrm{P}<0.01)$. (Table 5) 
Table 4: Bivariate analysis of socio-demographic, socio-economic factors and herbal medicine use among HIV positive patients on HAART

\begin{tabular}{|c|c|c|c|c|c|c|c|c|c|}
\hline \multirow[t]{3}{*}{ Variable } & & \multirow{2}{*}{\multicolumn{6}{|c|}{$\begin{array}{l}\text { Herbal Use } \\
\text { No }\end{array}$}} & \multirow{3}{*}{\multicolumn{2}{|c|}{ Test Stat, P- value }} \\
\hline & & & & & & & & & \\
\hline & & \multirow{2}{*}{$\begin{array}{l}\text { No. } \\
115\end{array}$} & \multirow{2}{*}{$\begin{array}{l}\mathrm{Col} \% \\
37.7\end{array}$} & No. & $C 01 / 0$ & & Corto & & \\
\hline \multirow[t]{2}{*}{ Gender $(n=361)$} & Male & & & 17 & 30.4 & 132 & 36.6 & \multicolumn{2}{|c|}{$\begin{array}{l}\text { Pearson } \quad \operatorname{chi} 2(1)= \\
1.1013, \operatorname{Pr}=0.294\end{array}$} \\
\hline & Female & 190 & 62.3 & 39 & 69.6 & 229 & 63.4 & & \\
\hline \multirow[t]{3}{*}{ Age $(n=355)$} & $<35$ & 87 & 29 & 12 & 21.8 & 99 & 27.9 & \multirow{3}{*}{$\begin{array}{l}\text { Pearson } \operatorname{chi} 2(2) \\
1.1921, \operatorname{Pr}=0.551\end{array}$} & $=$ \\
\hline & $35-44$ & 99 & 33 & 20 & 36.4 & 119 & 33.5 & & \\
\hline & $45+$ & 114 & 38 & 23 & 41.8 & 137 & 38.6 & & \\
\hline \multirow{2}{*}{$\begin{array}{l}\text { Marital } \\
(\mathrm{n}=331)\end{array}$} & Not Married & 107 & 38.1 & 20 & 40 & 127 & 38.4 & \multirow{2}{*}{$\begin{array}{l}\text { Pearson chi2(1) } \\
0.0663, \operatorname{Pr}=0.797\end{array}$} & $=$ \\
\hline & Married & 174 & 61.9 & 30 & 60 & 204 & 61.6 & & \\
\hline \multirow[t]{3}{*}{ Education $(n=360)$} & Primary or less & 68 & 22.4 & 10 & 17.9 & 78 & 21.7 & \multirow{3}{*}{$\begin{array}{l}\text { Pearson } \quad \text { chi2(2) } \\
0.7427, \operatorname{Pr}=0.690\end{array}$} & $=$ \\
\hline & Secondary & 136 & 44.7 & 25 & 44.6 & 161 & 44.7 & & \\
\hline & Tertiary & 100 & 32.9 & 21 & 37.5 & 121 & 33.6 & & \\
\hline \multirow{3}{*}{$\begin{array}{l}\text { Employment } \\
(\mathrm{n}=344)\end{array}$} & Unemployed & 57 & 19.7 & 12 & 22.2 & 69 & 20.1 & \multirow{3}{*}{$\begin{array}{l}\text { Pearson } \quad \text { chi2(2) } \\
0.2006, \operatorname{Pr}=0.905\end{array}$} & $=$ \\
\hline & Employed & 142 & 49 & 26 & 48.1 & 168 & 48.8 & & \\
\hline & $\begin{array}{l}\text { Private } \\
\text { Business }\end{array}$ & 91 & 31.4 & 16 & 29.6 & 107 & 31.1 & & \\
\hline \multirow[t]{3}{*}{ Income $(n=282)$} & $<20000$ & 90 & 37.3 & 16 & 39 & 106 & 37.6 & \multirow{3}{*}{$\begin{array}{l}\text { Pearson chi2(2) } \\
0.8298, \operatorname{Pr}=0.660\end{array}$} & $=$ \\
\hline & 20000-39999 & 76 & 31.5 & 15 & 36.6 & 91 & 32.3 & & \\
\hline & $\begin{array}{l}40000 \quad \text { and } \\
\text { above }\end{array}$ & 75 & 31.1 & 10 & 24.4 & 85 & 30.1 & & \\
\hline
\end{tabular}

Table 5: Bivariate analysis of adherence, side effects, HAART start factors and herbal medicine use among HIV positive patients on HAART

\begin{tabular}{|c|c|c|c|c|c|c|c|c|}
\hline \multirow[t]{3}{*}{ Variable } & \multicolumn{7}{|l|}{ Herbal Use } & \multirow[t]{3}{*}{ Test Stat, P- value } \\
\hline & \multicolumn{3}{|l|}{ No } & \multicolumn{2}{|l|}{ Yes } & \multicolumn{2}{|c|}{ Total } & \\
\hline & No. & & $\mathrm{Col} \%$ & No. & $\mathrm{Col} \%$ & No. & $\mathrm{Col} \%$ & \\
\hline \multirow[t]{4}{*}{$\begin{array}{l}\text { Duration in years between HIV } \\
\text { diagnosis and HAART } \\
(\mathrm{n}=357)\end{array}$} & $\begin{array}{l}\text { Less than } \\
\text { 3years }\end{array}$ & 48 & 15.9 & 4 & 7.3 & 52 & 14.6 & \multirow[t]{4}{*}{$\begin{array}{l}\text { Pearson } \operatorname{chi} 2(3)=9.0929 \\
\operatorname{Pr}=0.028\end{array}$} \\
\hline & $3-5$ & 58 & 19.2 & 12 & 21.8 & 70 & 19.6 & \\
\hline & $6-8$ & 97 & 32.1 & 11 & 20 & 108 & 30.3 & \\
\hline & $\begin{array}{l}\text { More than } 9 \\
\text { years }\end{array}$ & 99 & 32.8 & 28 & 50.9 & 127 & 35.6 & \\
\hline \multirow[t]{2}{*}{ Adherence $(n=361)$} & Good / Fair & 191 & 62.7 & 24 & 42.9 & 215 & 59.5 & \multirow{2}{*}{$\begin{array}{l}\text { Pearson } \operatorname{chi} 2(2)=7.6742, \\
\operatorname{Pr}=0.006\end{array}$} \\
\hline & Poor & 114 & 37.4 & 32 & 57.1 & 146 & 40.4 & \\
\hline \multirow[t]{2}{*}{ Side effects after HAART start $(n=360)$} & No & 125 & 41.1 & 11 & 19.6 & 136 & 37.8 & \multirow[t]{2}{*}{$\begin{array}{l}\text { Pearson } \operatorname{chi} 2(1)=9.2783 \\
\operatorname{Pr}=0.002\end{array}$} \\
\hline & Yes & 179 & 58.9 & 45 & 80.4 & 224 & 62.2 & \\
\hline
\end{tabular}




\subsection{Multivariate analysis of factors associated with herbal medicine use among HIV positive patients taking HAART}

The factors that were independently associated with herbal medicine use were a more than 9 years' duration between HIV diagnosis and HAART start, poor adherence to HAART and side effects after HAART start. Patients who were started HAART more than 9 years' after HIV diagnosis were four times more likely to use herbal medicine as compared to those who were started HAART within 3 years of HIV diagnosis. Patients who had poor adherence to HAART had higher odds of using herbal medicine. Patients who had experienced side effects after HAART start were three times more likely to have used herbal medicine. (Table 6).

Table 6: Multivariate model for factors associated with herbal medicine use among HIV positive patients taking HAART

\begin{tabular}{|c|c|c|c|}
\hline Variable & & OR $(95 \% \mathrm{CI})$ & $\mathrm{P}$-value \\
\hline \multicolumn{4}{|c|}{$\begin{array}{l}\text { Duration in years between HIV } \\
\text { diagnosis and HAART start }\end{array}$} \\
\hline & 3-5 years & $2.692(0.761-9.561)$ & 0.087 \\
\hline & $6-8$ years & $1.673(0.445-5.608)$ & 0.392 \\
\hline & More than 9 years & $4.631(1.283-12.045)$ & 0.009 \\
\hline \multirow[t]{2}{*}{ Adherence } & Good/ Fair & Ref & \\
\hline & Poor & $2.84(1.55-7.81)$ & 0.027 \\
\hline \multirow[t]{2}{*}{$\begin{array}{l}\text { Side effects after } \\
\text { HAART start }\end{array}$} & $\begin{array}{l}\text { Didn't experience } \\
\text { effects }\end{array}$ & $\mathrm{e}_{\text {Ref }}$ & \\
\hline & Experienced side e & $3.402(2.015-4.165)$ & 0.031 \\
\hline
\end{tabular}

\subsection{Socio-demographic, economic factors and occurrence of adverse drug reaction among HIV positive patients taking HAART.}

The study established that $62 \%$ of HIV positive patient experienced adverse drug reactions after HAART start. A higher proportion of males had side effects as compared to females, $84(62.7 \%)$ vs. 144 (61.5\%), without statistical significance, $p=0.827$. There was no significant difference in proportion with side effects by marital status, $p=$ 0.845 . Similarly, there was no difference in proportion with side effects by education level and income level, $p$ $=0.205$ and $\mathrm{p}=0.204$ respectively. There was a higher proportion of those employed with side effects, $114(52.5 \%)$ vs. $56(41.8 \%), p=0.147$, without any statistical significance. (Table 7). 
Table 7: Bivariate analysis of socio-demographic, socio-economic factors and occurrence of adverse drug reactions among HIV positive patients on HAART

\begin{tabular}{|c|c|c|c|c|c|c|c|c|c|}
\hline \multirow[b]{3}{*}{ Gender $(n-368)$} & \multirow[t]{2}{*}{ Side Effects } & \multicolumn{2}{|c|}{ No $(n=140)$} & \multicolumn{2}{|c|}{ Yes $(n=228)$} & \multicolumn{2}{|c|}{ Total $(n=368)$} & \multicolumn{2}{|l|}{ Test statistic, $\mathrm{P}$ value } \\
\hline & & No. & $\mathrm{Col} \%$ & No. & $\mathrm{Col} \%$ & No. & $\mathrm{Col} \%$ & & \\
\hline & & & & & & & & Pearson chi2(1) & $=$ \\
\hline & Male & 50 & 35.7 & 84 & 36.8 & 134 & 36.4 & $0.0477, \operatorname{Pr}=0.827$ & \\
\hline \multirow{4}{*}{ Age $(n=360)$} & Female & 90 & 64.3 & 144 & 63.2 & 234 & 63.6 & & \\
\hline & & & & & & & & Pearson chi2(2) & $=$ \\
\hline & $<35$ & 32 & 23.2 & 69 & 31.1 & 101 & 28.1 & $2.8606, \operatorname{Pr}=0.239$ & \\
\hline & $35-44$ & 51 & 37 & 69 & 31.1 & 120 & 33.3 & & \\
\hline \multirow{3}{*}{$\begin{array}{l}\text { Marital status } \\
(\mathrm{n}=337)\end{array}$} & $45+$ & 55 & 39.9 & 84 & 37.8 & 139 & 38.6 & & \\
\hline & & & & 79 & & & 356 & Pearson chi2(2) & $=$ \\
\hline & Not Miarried & 51 & 39.2 & 19 & 38.2 & 130 & 53.0 & & \\
\hline \multirow{4}{*}{$\begin{array}{l}\text { Education Level } \\
(\mathrm{n}=366)\end{array}$} & Married & 79 & 60.8 & 128 & 61.8 & 207 & 61.4 & & \\
\hline & Primary or less & 37 & 26.6 & 44 & 19.4 & 81 & 22.1 & $\begin{array}{l}\text { Pearson chi2(2) } \\
3.1731, \operatorname{Pr}=0.205\end{array}$ & $=$ \\
\hline & Secondary & 61 & 43.9 & 101 & 44.5 & 162 & 44.3 & & \\
\hline & Tertiary & 41 & 29.5 & 82 & 36.1 & 123 & 33.6 & & \\
\hline \multirow{4}{*}{$\begin{array}{l}\text { Employment } \\
\text { Status }(\mathrm{n}=351)\end{array}$} & & & & & & & & Pearson chi2(2) & $=$ \\
\hline & Unemployed & 30 & 22.4 & 40 & 18.4 & 70 & 19.9 & $3.8313, \operatorname{Pr}=0.147$ & \\
\hline & Employed & 56 & 41.8 & 114 & 52.5 & 170 & 48.4 & & \\
\hline & Private business & 48 & 35.8 & 63 & 29 & 111 & 31.6 & & \\
\hline \multirow[t]{3}{*}{$\begin{array}{l}\text { Income } \\
(\mathrm{n}=288)\end{array}$} & $<20000$ & 39 & 36.1 & 67 & 37.2 & 106 & 36.8 & $\begin{array}{l}\text { Pearson chi2(2) } \\
\text { 3.1766, } \operatorname{Pr}=0.204\end{array}$ & $=$ \\
\hline & 20000-39999 & 31 & 28.7 & 66 & 36.7 & 97 & 33.7 & & \\
\hline & $\begin{array}{l}40000 \quad \text { and } \\
\text { above }\end{array}$ & 38 & 35.2 & 47 & 26.1 & 85 & 29.5 & & \\
\hline
\end{tabular}

\subsection{Bivariate analysis of adherence, HAART start factors and occurrence of adverse drug reactions among HIV positive patients on HAART}

There was a significant difference in proportion of those with side effects by duration in years between HIV diagnosis and HAART start, $\mathrm{p}<0.01$. Majority of those who had poor adherence had significantly higher proportion of side effects as compared those had good/fair adherence, 104 (45.65) vs. $45(32.1 \%), \mathrm{p}=0.011$. (Table 8). 
Table 8: Bivariate analysis of adherence, HAART start factors and occurrence of adverse drug reactions among HIV positive patients on HAART

\begin{tabular}{|c|c|c|c|c|c|c|c|c|}
\hline & Side Effects & No ( & 40) & Yes & 28) & Tota & 368) & \multirow[t]{2}{*}{ Test statistic, $\mathrm{P}$ value } \\
\hline & & No. & $\mathrm{Col} \%$ & No. & $\mathrm{Col} \%$ & No. & $\mathrm{Col} \%$ & \\
\hline \multirow[t]{3}{*}{$\begin{array}{l}\text { Duration in years } \\
\text { between } \\
\text { HIV diagnosis } \\
\text { and HAART } \\
(\mathrm{n}=364)\end{array}$} & $<3(\mathrm{n}=54)$ & 11 & 8 & 43 & 18.9 & 54 & 14.8 & $\begin{array}{l}\text { Pearson chi2(3) }= \\
12.2605, \operatorname{Pr}=0.007\end{array}$ \\
\hline & $3-5(n=69)$ & 21 & 15.3 & 48 & 21.1 & 69 & 19 & \\
\hline & $6-8(n=110)$ & 46 & 33.6 & 64 & 28.2 & 110 & 30.2 & \\
\hline \multirow[t]{2}{*}{$\begin{array}{l}\text { Adherence } \\
(\mathrm{n}=368)\end{array}$} & $\begin{array}{l}>9(n=131) \\
\text { Good/Fair } \\
(n=219)\end{array}$ & 59 & 43.1 & 72 & 31.7 & 131 & 59.5 & $\begin{array}{l}\text { Pearson chi2(1) }= \\
6.5327, \operatorname{Pr}=0.011\end{array}$ \\
\hline & Poor $(n=149)$ & 45 & 32.1 & 104 & 45.6 & 149 & 40.5 & \\
\hline
\end{tabular}

3.9 Logistic regression of socio-demographic, economic factors, HAART start and occurrence of adverse drug reaction among HIV positive patients taking HAART.

On univariable analysis, females had a slightly lowered odds of having a side effect $\mathrm{OR}=0.9595 \% \mathrm{CI}(0.62-1.48)$, $\mathrm{p}=0.827$ without statistical significance.

Those started HAART 6-8 years after HIV diagnosis, OR=0.36 95\% CI (0.17-0.76), and those started more than 9 years $\mathrm{OR}=0.3195 \% \mathrm{CI}(0.15-0.66)$ had statistically significant lower odds of side effects compared to those started HAART less than 3 years after diagnosis, $\mathrm{p}=0.008$ and $\mathrm{p}=0.002$ respectively. Those with poor adherence compared to those with good or fair adherence, had statistically significant higher odds of side effects, $\mathrm{OR}=1.77$ 95\% CI (1.14-2.75).

Multivariable model included all the variables. On multivariable analysis, patients started HAART 6-8 years after HIV diagnosis, $\mathrm{OR}=0.3595 \% \mathrm{CI}(0.13-0.91)$, and more than 9 years $\mathrm{OR}=0.2795 \% \mathrm{CI}(0.1-0.73)$ had statistically significant lower odds of side effects compared to those started HAART less than 3 years, $p=0.032$ and $p=0.009$ respectively. Patients with poor adherence compared to those with good or fair adherence, had statistically significant higher odds of side effects, $\mathrm{OR}=2.3895 \% \mathrm{CI}(1.33-4.27), \mathrm{p}=0.004$. (Table 9). 
Table 9: Multivariate model for factors associated with adverse drug reactions among patients on HAART

\begin{tabular}{|c|c|c|c|c|c|}
\hline & Side Effects (Yes vs. No) & Univariable OR & $\mathrm{P}$ value & Multivariable OR & P value \\
\hline Gender & Male & Ref & & Ref & \\
\hline \multirow{3}{*}{ Age Category } & Female & $0.95(0.62-1.48)$ & 0.827 & $1.28(0.72-2.3)$ & 0.403 \\
\hline & $<35$ & Ref & & Ref & \\
\hline & $35-44$ & $0.63(0.36-1.09)$ & 0.099 & $1.06(0.49-2.31)$ & 0.883 \\
\hline \multirow{3}{*}{ Marital status } & $45+$ & $0.71(0.41-1.22)$ & 0.210 & $1.19(0.54-2.6)$ & 0.668 \\
\hline & Not Married & Ref & & Ref & \\
\hline & Married & $1.05(0.67-1.64)$ & 0.845 & $1.17(0.54-2.17)$ & 0.639 \\
\hline \multirow{2}{*}{$\begin{array}{l}\text { Education } \\
\text { Level }\end{array}$} & Primary school or less & Ref & & Ref & \\
\hline & Secondary school & $1.39(0.81-2.39)$ & 0.230 & $1.38(0.62-3.05)$ & 0.428 \\
\hline \multirow{3}{*}{$\begin{array}{l}\text { Employment } \\
\text { Status }\end{array}$} & Tertiary & $1.68(0.95-2.99)$ & 0.077 & $2.43(0.94-6.25)$ & 0.0663 \\
\hline & Unemployed & Ref & & Ref & \\
\hline & Employed & $1.53(0.86-2.7)$ & 0.147 & $2.26(0.24-21.6)$ & 0.480 \\
\hline \multirow{3}{*}{ Income level } & Private business & $0.98(0.54-1.8)$ & 0.959 & $1.73(0.18-16.7)$ & 0.635 \\
\hline & $<20000$ & Ref & & Ref & \\
\hline & 20000-39999 & $1.24(0.69-2.22)$ & 0.469 & $1.32(0.63-2.75)$ & 0.463 \\
\hline \multirow{4}{*}{$\begin{array}{l}\text { Duration in } \\
\text { years between }\end{array}$} & 40000 and above & $0.72(0.4-1.29)$ & 0.268 & $0.57(0.26-1.26)$ & 0.164 \\
\hline & $<3$ & Ref & & Ref & \\
\hline & $3-5$ & $0.59(0.25-1.35)$ & 0.209 & $0.63(0.22-1.83)$ & 0.399 \\
\hline & $6-8$ & $0.36(0.17-0.76)$ & 0.008 & $0.35(0.13-0.91)$ & 0.032 \\
\hline \multirow{3}{*}{ Adherence } & $>9$ & $0.31(0.15-0.66)$ & 0.002 & $0.27(0.1-0.73)$ & 0.009 \\
\hline & Good/Fair & Ref & & Ref & \\
\hline & Poor & $1.77(1.14-2.75)$ & 0.011 & $2.38(1.33-4.27)$ & 0.004 \\
\hline
\end{tabular}

\subsection{Benefits of HAART known by HIV positive patients on HAART}

The benefits of taking HAART were known by 359(98.4\%) participants with 6(1.6\%) not knowing any benefit of taking HAART. The benefits known to study participants were to suppress viral load, improve or prolong life and to boost immunity among others. (Figure 1). 


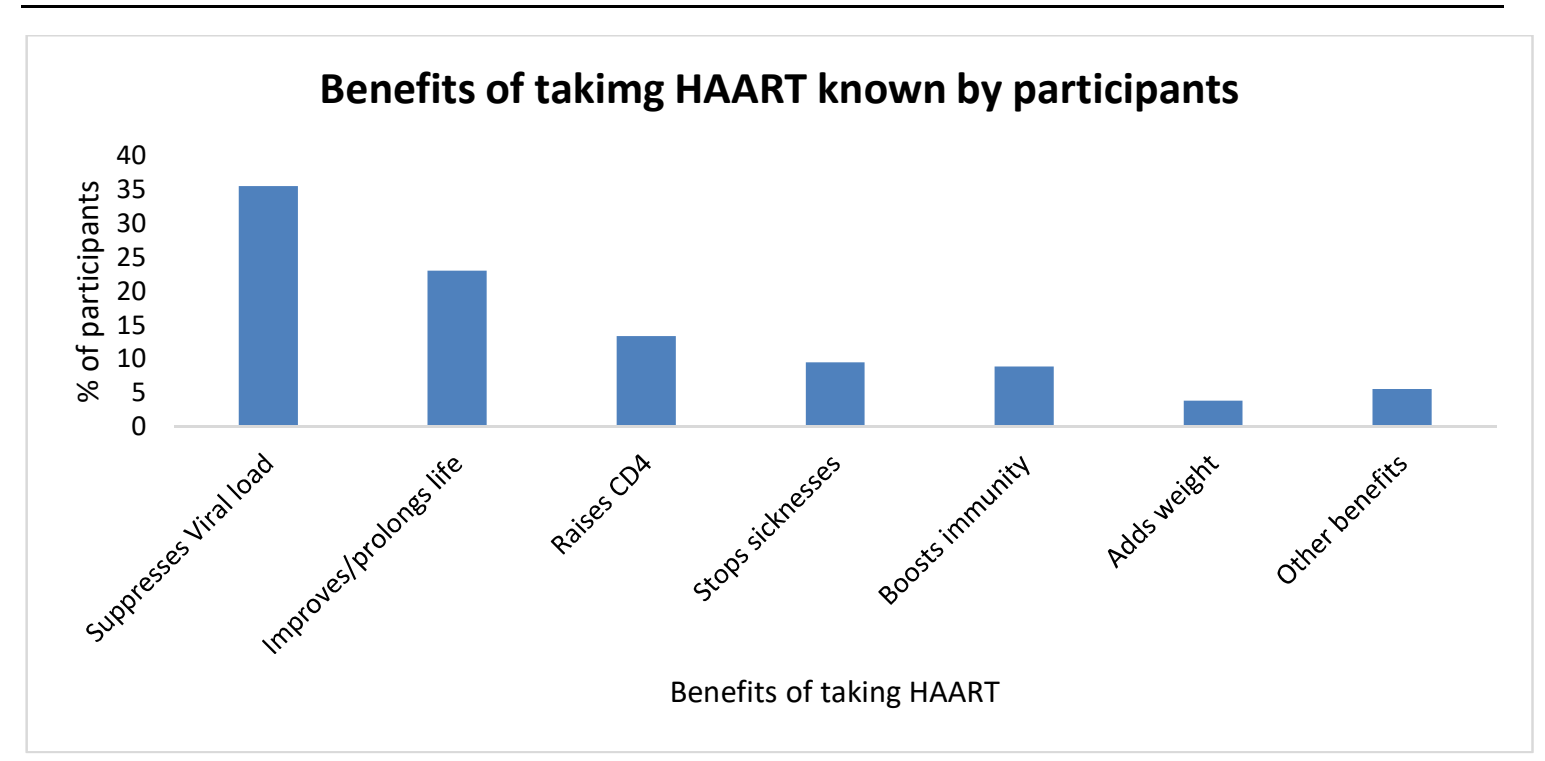

Figure 1: Benefits of taking HAART known by HIV positive patients taking HAART

\subsection{Side effects experienced by HIV positive patients on HAART}

After HAART start, $62 \%$ of the patients experienced side effects. The side effects experienced were nausea and vomiting 47(20.6\%), drowsiness 42(18.4\%), dizziness 31(13.6\%), hallucinations and joint pains 39(17.1\%), skin rashes $13(5.7 \%)$, weight loss $17(7.5 \%)$ and night mares $15(6.6 \%)$ and other side effects $24(10.5 \%)$. (Figure 2)

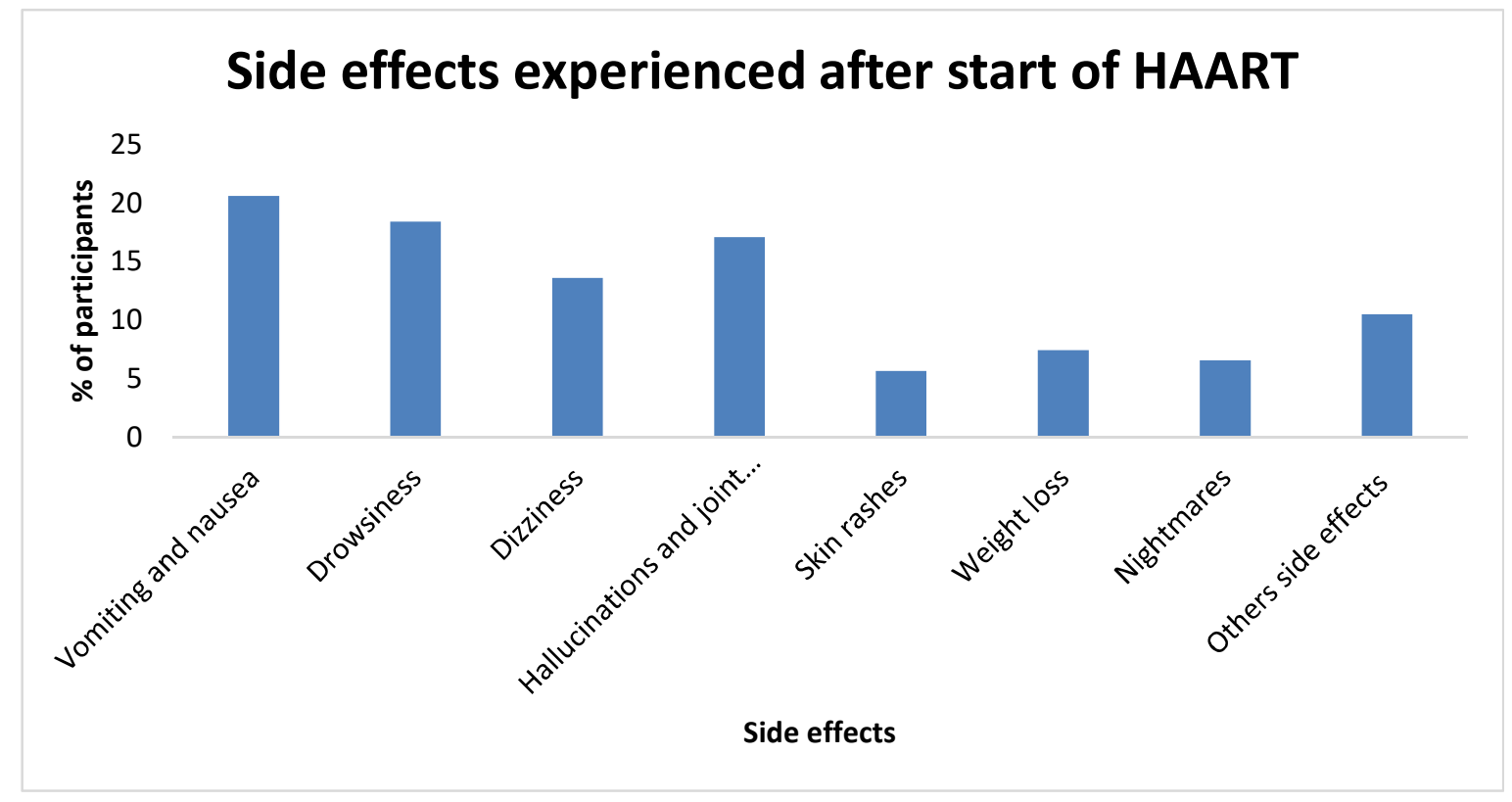

Figure 2: Side effects experienced participants After HAART start

\subsection{Types of herbal medicine used by HIV positive patients on HAART}

The types of herbal medicine used by HIV positive patients on HAART included Loliondo 20(35.7\%), aloevera $12(21.4 \%)$, marijuana $9(16 \%)$ while $15(26.8 \%)$ users did not know the names of herbal medicines they used. (Figure 3) 


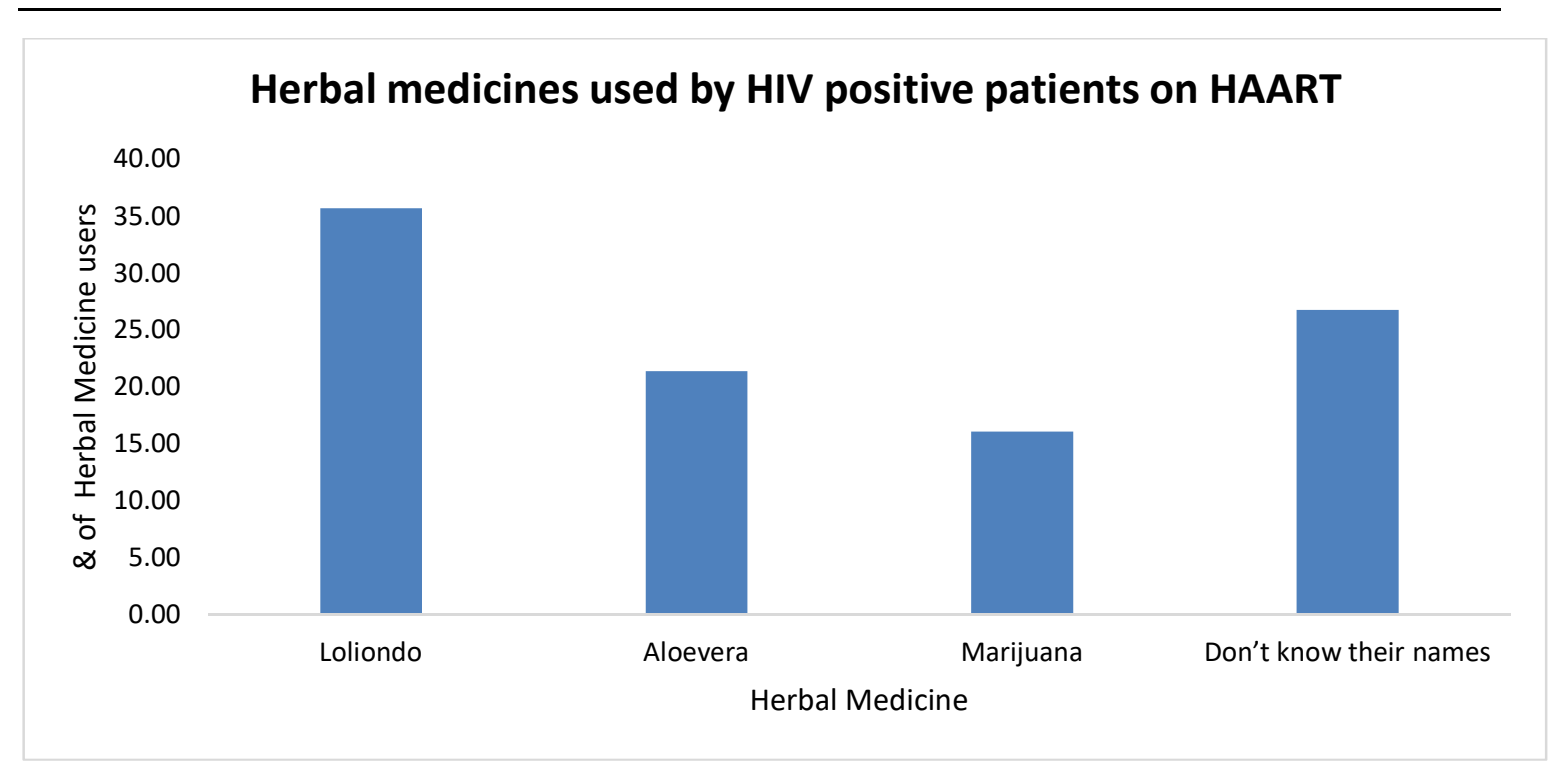

Figure 3: Types of herbal medicines used by HIV positive patients on HAART

3.13 Routes of administration of herbal products used by HIV positive patients on HAART

The routes of administration of herbal products by HIV positive patients on HAART were oral 100\%, topical 7\%, inhalation at $7 \%$, rectal $2 \%$ and vaginal $0 \%$. (Figure 4 ).

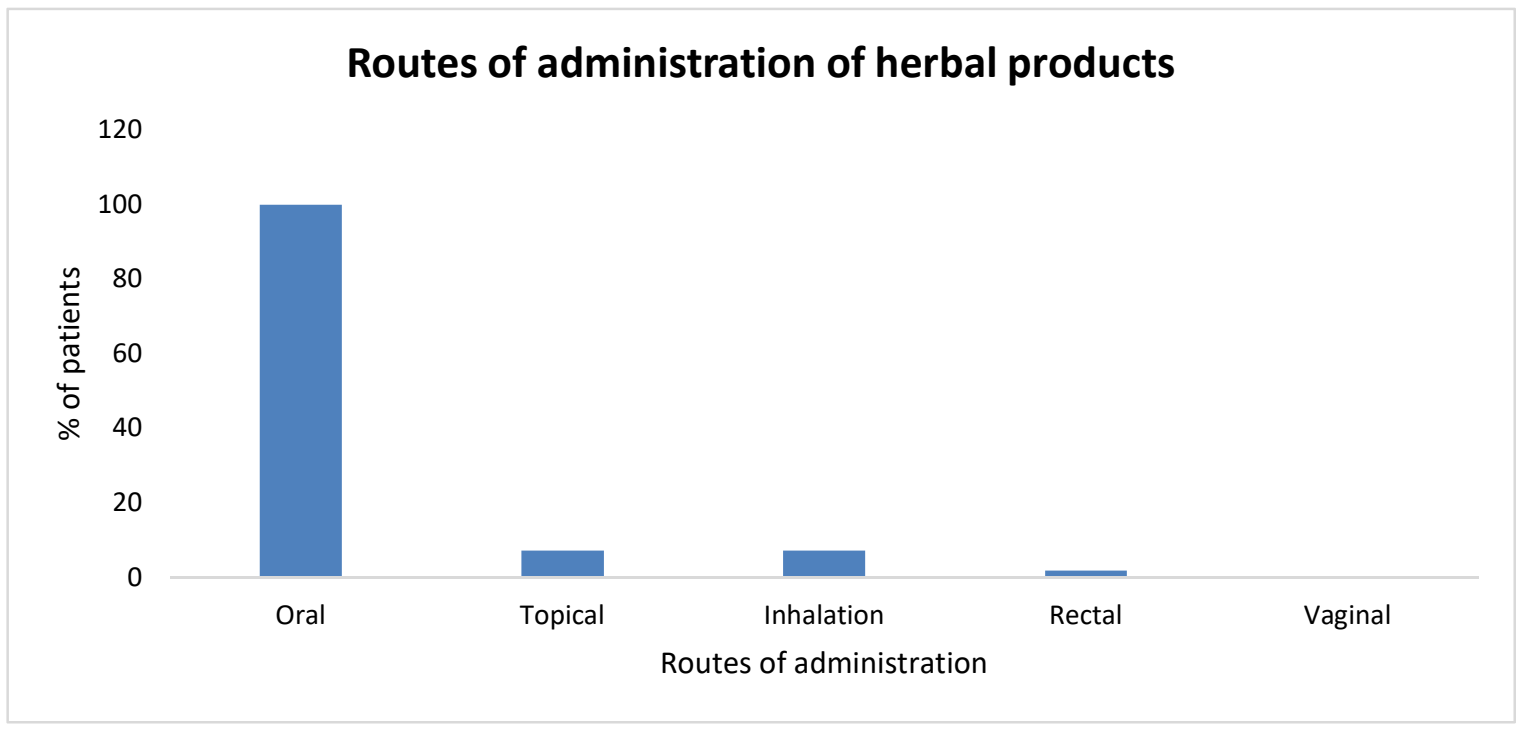

Figure 4: Routes of administration of herbal products used by HIV positive patients on HAART

\subsection{Sources of education programs on herbal medicine for HIV treatment}

The sources of education programs on herbal medicine for HIV treatment were TV (79\%), radio $(72.4 \%)$, newspaper (35.7\%), magazine (25.5\%), books (23.8\%), internet (38\%), market place (87.8\%), religious gathering $(20.3 \%)$. (Figure 5). 


\section{Sources of education programs on Herbal medicine use for HIV treatment}

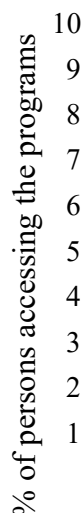

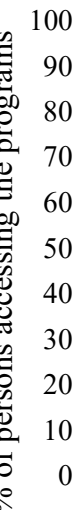
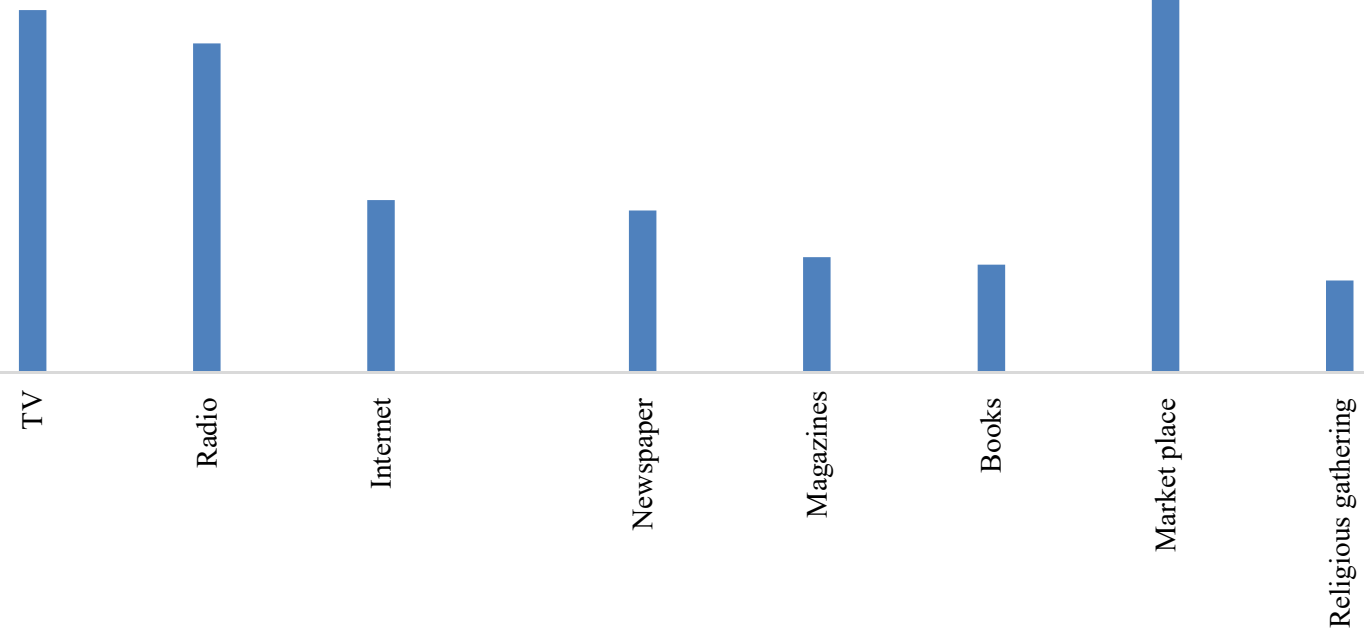

Sources of education programs

Figure 5: Sources of education programs on herbal medicine for HIV treatment

\subsection{Money spent per month on herbal medicine by HIV positive patients on HAART}

The study established that approximately $45 \%$ of the participants who use herbal medicine spent less than Ksh1,000/= per month on herbal medicines while about $15 \%$ spent over Ksh5,000 per month on herbal medicine. (Figure 6).

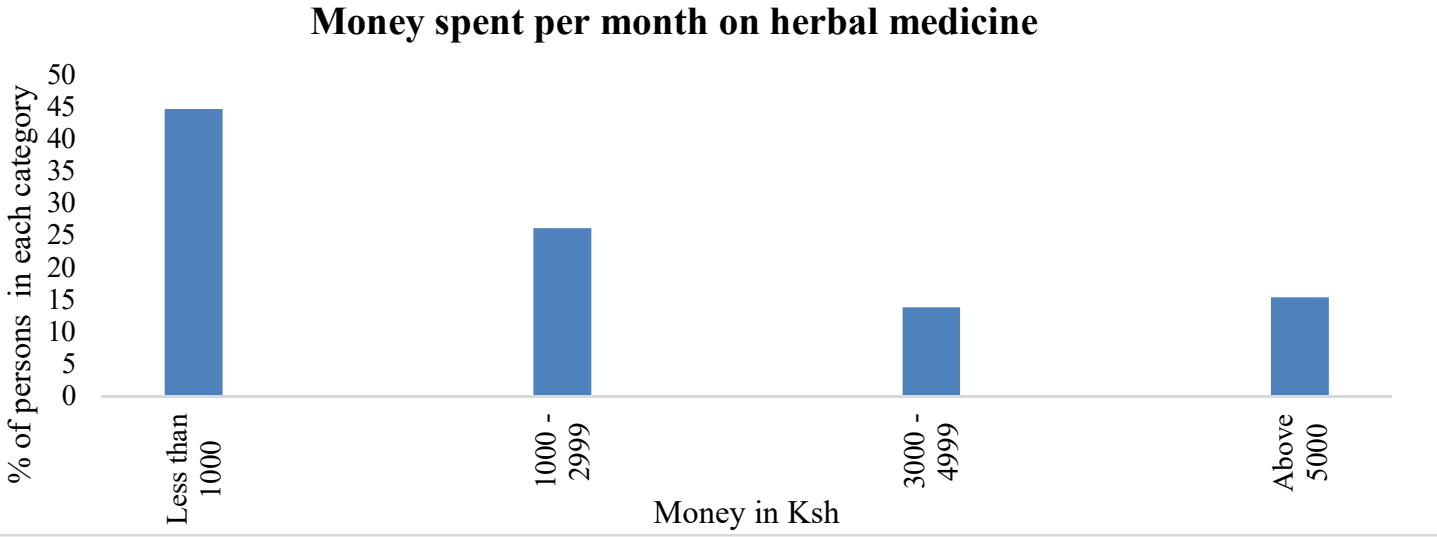

Figure 6: Money spent per month on herbal medicine 


\subsection{Reasons for preferring herbal medicine, sources and disclosure of herbal medicine use by HIV positive patients on HAART}

The reasons for preferring herbal medicine is that it is easily accessible (97.79\%), more acceptable (97.26\%) and also because herbal medicine is cheap (92.86\%). The sources of herbal medicine were the herbalist $(92.59 \%)$, garden $(87.06 \%)$, friends $(80.77 \%)$, pharmacy $(50.00 \%)$. With regard to disclosure of herbal medicine use to health care workers, $67.3 \%$ of the participants who used herbal medicine had never disclosed their herbal medicine use to any clinician at the comprehensive care Centre. After herbal medicine use, $44.2 \%$ of HIV positive patients on HAART felt that their health improved. The study established that $16.1 \%$ of HIV positive patients on HAART who used herbal medicine had ever stopped taking HAART to take herbal medicine.

\subsection{Discussion}

\subsection{Prevalence of herbal medicine use among HIV positive patients on HAART}

The study established that $15.51 \%$ of the study participants used herbal medicine together with HAART. These finding concur to those of Babb et al (2007) that in Africa many HIV patients use traditional herbal medicine for primary health care or management of side effects. The prevalence of herbal medicine use among HIV positive patients on antiretroviral therapy in this study was lower than in Uganda (33.7\%) as reported by Namuddu et al (2011). This could be attributed to patient education by health care providers that discourage the patients from taking herbal remedies while on HAART. A study by Bepe et al (2011) established that 54\% of the patients were taking antiretroviral drugs together with one or more traditional herbal remedies. In this study more $17 \%$ of females and $12.9 \%$ of males reported to be using herbal medicines with HAART. The study findings are consistent with the findings of Agnoletto et al (2006) that showed CAM use was more common among females than males with an odds ratio of $1.6 ; 95 \%$ confidence interval.

\subsection{Socio-demographic, economic factors and herbal medicine use among HIV positive patients taking HAART.}

In this study, the prevalence of herbal medicine use among the women (17\%) was higher than that in men (12.9\%), but this was not statistically significant. A study by Gore-Felton et al (2003) established that women were four times more likely to use alternative therapies than men. Furler et al (2003) observed that Complementary and alternative medicine use in Canadian patients with HIV was extremely common, with higher use among women. The study established that the prevalence of herbal medicine use among HIV positive patients on HAART who had attained tertiary education (17.4\%) was higher than for those who had attained secondary school education $(15.5 \%)$ and those who had primary school education $(12.8 \%)$, but this was not statistically significant. These results compare well with what was reported by Bica et al (2003) that users of ingested complementary therapies were more likely to be at least high school educated.

In this study, there was no statistically significant association between employment status and herbal medicine use. This is unlike what was observed by Otang et al (2011) who reported that the proportion of unemployed HIV/AIDS patients who used herbs for symptom management was significantly higher than that of the employed.

\subsection{Adherence and herbal medicine use among HIV positive patients taking HAART.}

The study established that herbal medicine use led to decreased adherence to HAART (P-value 0.006). These findings are consistent with the findings of Peltzer et al (2010) that the use of herbal treatments was associated with ARV non-adherence. The findings of this study are consistent with those of Namuddu et al (2011) who observed that Participants with HAART adherence levels $>95 \%$ were less likely to use traditional herbal medicine.

\subsection{The association between herbal medicine use and occurrence of adverse drug reactions among HIV positive patients taking HAART.}

This study established that patients who used herbal medicine with HAART were more likely to experience adverse drug reactions, $p$-value $<0.01$. Bepe et al (2011) observed that abdominal pain (odds ratio $=3.0$, $p$-value $<0.01)$ and rash (odds ratio $=2.5, \mathrm{p}$-value $<0.02$ ) are the adverse events significantly associated with herbal drug 
use during antiretroviral therapy. The findings of this study are unlike what was observed by Mudzviti et al (2012) who reported that Patients who used the indigenous herbs Musakavakadzi and Peltoforum africanum were less likely to develop adverse drug reactions compared to patients who did not use the herbs.

\subsection{Factors associated with occurrence of adverse drug reactions among HIV positive patients on HAART.}

This study established that patients with poor adherence had statistically significant higher odds of side effects compared to those with good or fair adherence, $, \mathrm{OR}=2.3895 \% \mathrm{CI}(1.33-4.27), \mathrm{p}=0.004$. these findings compare well with those of Rajesh et al (2012) who observed the occurrence of ADRs to antiretroviral therapy was significantly associated and significantly predicted with the percentage of adherence being less than $80 \%(P<$ $0.001)$.

\subsection{Side effects experienced by HIV positive patients on HAART}

In this study $62 \%$ of the patients experienced side effects since they started using ARVs. These findings compare well with the findings of Tadesse et al (2014) that $89.8 \%$ of the study participants experienced at least one adverse drug reaction. A study by Masenyetse et al (2015) established that $37 \%$ of the patients on Highly active antiretroviral drugs experienced adverse drug reactions. The findings of this study correspond with those of Mudzviti et al (2012) who observed that $70.4 \%$ of the patients experienced at least one ADR during ART treatment.

\subsection{Types of herbal medicine used by HIV positive patients on HAART}

The types of herbal medicine used by HIV positive patients on HAART included Loliondo 20(35.7\%), aloe Vera $12(21.4 \%)$, marijuana $9(16 \%)$ while $15(26.8 \%)$ users did not know the names of herbal medicines they used. The choice of herbal medicine could be attributed to availability, geographical accessibility and traditional believes. A study by Hasan et al., (2010) reported that among the types of Complementary and alternative medicine used by Malaysian patients with HIV/AIDS, 33.8\% of the patients used herbal products. Traditional Chinese medicine and traditional Malay medicine were the most frequently used types of herbal medicine. The study findings are consistent with those of Klassen et al (2007) who reported that some patients didn't know the specific names of the traditional herbal medicines they used.

\subsection{Sources of education programs on herbal medicine for HIV treatment}

The study established that the sources of education programs on herbal medicine for HIV treatment were TV (79\%), radio $(72.4 \%)$, newspaper $(35.7 \%)$, magazine $(25.5 \%)$, books $(23.8 \%)$, internet $(38 \%)$, market place $(87.8 \%)$, religious gathering $(20.3 \%)$. These findings are consistent with those of Hasan et al., (2010) where most of the patients reported using complementary and alternative medicine upon the influence of family members and some of them were also seeking information from the mass media after being infected with HIV/AIDS.

\subsection{Reasons for preferring herbal medicine, sources and disclosure of herbal medicine use by HIV positive patients on HAART}

The study established reasons for preferring herbal medicine is that it is easily accessible (97.79\%), more acceptable $(97.26 \%)$ and also because herbal medicine is cheap $(92.86 \%)$. These findings are consistent with the findings of Lambert et al (2011) that in Kenya, patients would opt for traditional herbal medicine because the traditional herbal medicine practitioners are more accessible than the trained doctors but also a large number of the rural poor, in particular, turn to traditional medical practitioners for certain conditions, when access to allopathic health workers is restricted. Klassen et al (2007) observed that the reasons for use of traditional herbal medicine were availability/abundance of THM, treatment effectiveness of THM, proximity of the herbs to the patient, familiarity with THM and affordability.

The study established that the largest source of herbal medicine for the patients was from herbalist (84.2\%) and the smallest source was from the pharmacy $(66.7 \%)$. These findings are in line with the findings of Namuddu et al (2011) that reported most of the herbs were obtained from registered herbalist and very few were obtained from pharmacies.

This study established that $44.2 \%$ of the patients who used herbal medicine felt that their health improved. These findings are consistent with the finding of Hasan et al (2010) that in Malysia 40\% of HIV positive CAM users felt that CAM improved their health status and none of them felt that their health had deteriorated due to CAM use. 
The study findings are consistent with those of Otang et al (2011) who reported that $32.5 \%$ patients disclosed that herbal medicine had a significant and great contribution towards the management of their signs/symptoms of opportunistic fungal infections.

The study established that $16.1 \%$ of HIV positive patients on HAART who used herbal medicine had ever stopped taking HAART to take herbal medicine. This is more than what was reported by Fairfield et al (1998) where the study established that $1.7 \%$ of the patients decided to take CAM only and not HAART for management of HIV infection.

This study established that $67.3 \%$ of the patients who used herbal medicine had never disclosed to the doctor or any health care worker at the CCC about their herbal medicine use. These findings are consistent with those of Peltzer et al (2008) who reported that HIV infected people don't like disclosing to the health care professionals about their CAM use. A study by Hasan et al (2010) reported that Despite the side-effects experienced by the study participants, about $68 \%$ and $84 \%$ of them did not disclose their complementary and alternative medicine use to doctors and pharmacists, respectively. Underlying reasons for nondisclosure were unnecessary to disclose, and that health care professionals never asked regarding complementary and alternative medicine use.

\subsection{Conclusions}

- The Prevalence of herbal medicine use among HIV positive patients on highly active antiretroviral therapy is $15.5 \%$.

- Herbal medicine use reduces adherence to HAART whereas $16.1 \%$ of HIV positive patients on HAART who also use herbal medicine actually stop taking HAART to take herbal medicine.

- Majority of HIV positive patients who use both HAART and herbal medicines (67.3\%) do not disclose their herbal medicine use to the health care workers at the CCC clinic.

- The biggest source of herbal drugs is from the herbalists with the smallest source being from the pharmacy.

- The main reasons why patients opt for herbal medicine is because herbal medicine is easily accessible, more acceptable and cheap.

- Media plays an important role by virtue of information that goes to patients about herbal medicine.

- The use of herbal drugs increases the chances of the patients experiencing adverse drug reactions once started on HAART.

\subsection{Recommendations}

1. The prevalence of herbal medicine use among HIV positive patients on HAART is high and therefore further investigations ought to be done to establish the safety, efficacy and drug interactions between herbal drugs commonly used by HIV positive patients and antiretroviral agents used for HIV/AIDS treatment

2. Herbal medicine use reduces adherence to HAART and therefore adherence being the greatest patientenabled predictor of treatment success should be reinforced at every clinic visit.

3. Majority of HIV positive patients who use both HAART and herbal medicines do not disclose their herbal medicine use to the health care workers at the CCC clinic and therefore the healthcare workers should routinely discuss with the patients on herbal medicine use at every clinic visit.

4. The biggest source of herbal drugs is from the herbalists and therefore the government should introduce a basic training program for herbalists and also put in place laws and regulations on the use and trade in herbal products to enhance patient safety.

5. There is need for the health sector to reduce barriers to universal access to conventional health care by ensuring easier accessibility, affordability and acceptability in the population.

6. Media plays an important role by virtue of information that goes to patients and therefore there is need for regulation of the media content about herbal medicine to protect patients from misleading information.

7. Concomitant use of herbal drugs with HAART has the potential for drug interactions and ADRs and therefore should be discussed routinely in HAART medication use counselling and patients screened thoroughly for ADRs for early identification and management. 


\section{References}

Agnoletto, V., Chiaffarino, F., Nasta, P., Rossi, R., \& Parazzini, F. (2006). Use of complementary and alternative medicine in HIV-infected subjects. Complement Ther Med, (3):193-199.

Aschwanden, C. (2001). Herbs for health, but how safe are they? Bulletin of the World Health Organization. https://www.ncbi.nlm.nih.gov/pmc/articles/PMC2566478.

Babb, D. A., Pemba, L., Seatlanyane, P., Charalambous, S., Churchyard, G. J., \& Grant, A. D. (2007). Use of traditional medicine by HIV-infected individuals in South Africa in the era of antiretroviral therapy. Psychol Health Med, 12(3):314-320.

Bepe, N., Madanhi, N., Mudzviti, T., Gavi, S., Maponga, C. C., \& Morse, G. D. (2011). The impact of herbal remedies on adverse effects and quality of life in HIV-infected individuals on antiretroviral therapy. Journal of Infection in Developing Countries, 5(1), 48-53.

Bica, I., Tang, A. M., Skinner, S., Spiegelman, D., Knox, T., Gorbach, S., Wilson, I. B. (2003) Use of complementary and alternative therapies by patients with human immunodeficiency virus disease in the era of highly active antiretroviral therapy. J Altern Complement Med 2003, 9(1):65-76.

Borg, W. R., \& Gall, M. D. (1989). Educational research. An introduction (5th ed.). White Plains, NY: Longman.

Cochran, W. G. (1977). Sampling techniques (3rd ed.). New York: John Wiley \& Sons.

Department of Health Operational Plan for Comprehensive HIV and AIDS Care, Management and Treatment for South Africa. (2007). http://www.info.gov.za/otherdocs/2003/aidsplan.pdf

Duggan, J., Peterson, W. S., \& Schutz, M. (2001). Use of complementary and alternative therapies in HIV-infected patients. AIDS Patient Care STDS 2001;15:159-167.

Durant, J., Clevenbergh, P., Garraffo, R., Halfon, P., Icard, S., Del Giudice, P., Montagne, N., Schapiro, J. M., Dellamonica, P. (2000). Importance of protease inhibitor plasma levels in HIV-infected patients treated with genotypic-guided therapy. Pharmacological data from the Viradapt Study. AIDS 14: 1333-133-9. https://www.ncbi.nlm.nih.gov/pubmed/10930147.

Eisenberg, D., Davis, R., Ettner, S., Appel, S., Wilkey, S., Van Rompay, M., and Kessler, R. (1998). Trends in alternative medicine use in the United States, 1990-1997. JAMA. 1998 Nov 11;280(18):1569-75.

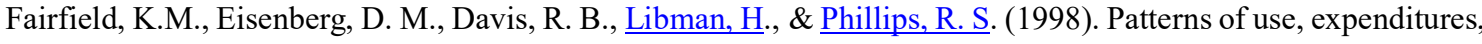
and perceived efficacy of complementary and alternative therapies in HIV-infected patients. JAMA 1998; 158:2257-2264.

UNAIDS. (2008). Fast facts about http://www.unaids.org/en/media/unaids/contentassets/dataimport/pub/factsheet/2008/20080519_fastfacts_hiv_en .pdf assessed on 18/06/2012

Fisher, A., Laing, J., \& Stoeckel, J. (1983). Guidelines for overcoming design problems in family planning operations research. Mediline.16 (2): 100-105

Furler, M. D., Einarson, T. R., Walmsley, S., Millson, M., \& Bendayan, R. (2004). Use of complementary and alternative medicine by HIV-infected outpatients in Ontario, Canada. AIDS Patient Care and STDs VOL. 17, NO. 4. https://doi.org/10.1089/108729103321619764

Gore-Felton, C., Vosvick, M., Power, R., Koopman, C., Ashton, E., Bachmann, M. H., Israelski, D., \& Spiegel, D. (2003). Alternative therapies: a common practice among men and women living with HIV. J Assoc Nurses AIDS Care 2003, 14(3):17-27.

Grace, N. N., Isaac, M. K., Peter, K. N., and Peter, O.O. (2010). Utilisation of priority traditional medicinal plants and local people's knowledge on their conservation status in arid lands of Kenya (Mwingi District). Journal of ethnobiology and ethnomedicine 2010; 6: 22. doi: 10.1186/1746-4269-6-22 PMCID: PMC2930631 
Hasan, S., Keong, S., Kwok, L., Ahmed, S., Ahmadi, K., Anwar, M. (2010). Reasons, Perceived Efficacy, and Factors Associated with Complementary and Alternative Medicine Use Among Malaysian Patients with HIV/AIDS. The journal of alternative and complementary medicine Volume 16, Number 11, 2010, pp. 1171-1176 Mary Ann Liebert, Inc. DOI: 10.1089/acm.2009.0657.

Hoareau, L.. \& Da Silva, E. J. (1999). Medicinal plants: a re-emerging health aid. Electronic Journal of Biotechnology, 1999, 2: 56-70. DOI: 10.2225/vol2-issue2-fulltext-2

Hsiao, A. F., Wong, M. D., Kanouse, D. E., Collins, R. L., Liu, H., Andersen, R. M., Gifford, A. L., McCutchan, A., Bozzette, S. A., \& Shapiro, M. F. (2003). Complementary and alternative medicine use and substitution for conventional therapy by HIV-infected patients. J Acquir Immune Defic Syndr 2003, 33(2):157-165.

Izzo, A. A. (2004). Herb-drug interactions: an overview of the clinical evidence. Fundamental Clinical Pharmacology.

Kenya AIDS indicator survey. (2007).

Kenya AIDS indicator survey. (2012).

Kenya Demographic and Health Survey

http://www.nacc.or.ke/nacc\%20downloads/kdhs_20082009_final_report.pdf

King, R., \& Homsy, J. (1997). Involving traditional healers in AIDS education and counselling in sub-Saharan Africa: a review. AIDS 11 (Suppl A): S217-S225.

Klassen, D., Kipp, W., Jhangri, G. S., \& Rubaale, T. (2007) Use of traditional herbal medicines by AIDS patients in Kabarole District, Western Uganda. Am J of Trop Med and Hyg 77: 757-763.

Korb-Savoldelli, V., Gillaizeau, F., Pouchot , J., Lenain, E., Postel-Vinay, N., Pierre-Franc., Durieux, P., \& Sabatier, B. (2012). Validation of a French Version of the 8-Item Morisky Medication Adherence Scale in Hypertensive Adults.

Kothari, C. R. (2004). Research Methodology: Methods and Techniques, New Delhi; New age International Ltd.

Lambert, J., Leonard, K., Mungai, G., Ogaja, E., Gatheru, G., Mirangi, T., Owara, J., Herbst, C., Ramana, G., \& Lemiere, C. (2011). The contribution of traditional herbal Medicine practitioners to Kenyan health care Delivery.

Lee, L. S., Andrade, A. S., \& Flexner, C. (2006). Interactions between Natural health products and antiretroviral drugs. Pharmacokinetic Pharmacodynamic Effects. Clin Infect Dis 2006, 43(8):1052-1059

Maina, N., Kagira, J. M., Achila, O., Karanja, S. M., \& Ngotho, M. (2013). Herbal medicines in Kenya: A review of the toxicity and quality control issues.

Malangu, N. (2007). Self-reported use of traditional, complementary and over-the-counter medicines by HIVinfected patients on antiretroviral therapy in Pretoria, South Africa. Afr. J. Trad. CAM. 4(3): 273-278.

Maponga, C., Chizanga, T., \& Morse, G. (2007). Traditional medicine use in HIV-infected patients in Harare. 4th IAS conference on HIV pathogenesis, treatment and prevention; Sydney, Australia; 22-25 Jul 2007

Mark, O. N., James, M. M., Adamson, L. L., Cyrus, G. W., Kipsengeret, B. K., Humphrey, F. K., Rahab, W. M., \& Wiliam, O. O. (2008). Ethnopharmacological survey of samburu district, Kenya.

Masenyetse, L. J., Manda, S. O., \& Mwambi, H. G. (2015). An assessment of adverse drug reactions among HIV positive patients receiving antiretroviral treatment in South Africa. AIDS Research and Therapy, 12, 6. http://doi.org/10.1186/s12981-015-0044-0

Mills, E., Foster, B. C., Van-Heeswijk, R., Phillips, E., Wilson, K., Leonard, B., Kosuge, K., Kanfer, I. (2005). Impact of African herbal medicine on antiretroviral metabolism. AIDS 19: 95-96.

Ministry of health Kenya, National AIDS Control Council (2014). Kenya HIV county profiles 
Morisky, E. D., Ang, A., Krousel-Wood, M., \& Ward, J. H. ( 2008). Predictive Validity of a Medication Adherence Measure in an Outpatient Settings.

Mudzviti, T., Maponga, C., Khoza, S., Qing, M., and Morse G. D. (2012). The Impact of Herbal Drug Use on Adverse Drug Reaction Profiles of Patients on Antiretroviral Therapy in Zimbabwe.

Mugenda, O.M and Mugenda, A.G. (2003). Research Methods. Quantitative and Qualitative.Approaches. Nairobi; African Centre for Technology Studies.

Mukherjee, P. K. (2002). Quality control of herbal drugs. Eastern publishers, Business Horizons, New Delhi, 2002, pp 1-37.

Namuddu, B., Kalyango, J., Karamagi, C., Mudiope, P., Sumba, S., Kalende, H., Wobudeya, E., Kigozi, B., \& Waako, P. (2011). Prevalence and factors associated with traditional herbal medicine use among patients on highly active antiretroviral therapy in Uganda. BMC Public Health 2011, 11:855 doi:10.1186/1471-2458-11-855

NASCOP. (2011). Guidelines for antiretroviral therapy in Kenya 4th edition. http://nascop.or.ke/library/ART\%20guidelines/Final\%20guidelines\%20re\%20print\%2011-09-2012.pdf

National Antiretroviral Treatment Guideline, National Department of Health South Africa (2004). (http://www.kznhealth.gov.za/arv/arv5.pdf) accessed on 8/4/2013.

Orodho, A. J. (2002). Techniques of Writing Research Proposals and Reports in Education and Social Sciences. Nairobi: Masola Publishers.

Orodho, A. J. (2005). Techniques of writing research proposals and reports in Educational and Social Sciences, ( $2^{\text {nd }}$ Edition $)$ Nairobi: Kaneja H.P Enterprises.

Otang, W. M., Grierson, D. S., \& Ndip, R. N. (2011). Prevalence, perceived benefits and effectiveness of herbal medicine in the management of symptoms of opportunistic fungal infections in HIV/AIDS patients in the Eastern Cape, South Africa. African Journal of Biotechnology Vol. 10(83), pp. 19458-19463, 21

Peltzer, K., Preez, N. F., Ramlagan, S., \& Fomundam, H. (2008). Use of traditional complementary and alternative medicine for HIV patients in KwaZulu-Natal, South Africa. BMC Public Health, 8, 255. http://doi.org/10.1186/1471-2458-8-255

Peltzer, K., Friend-du Preez, N., Ramlagan, S., Fomundam, H., \& Anderson, J. (2010). Traditional Complementary and Alternative Medicine and Antiretroviral Treatment Adherence Among HIV Patients in Kwazulu-Natal, South Africa. African Journal of Traditional, Complementary, and Alternative Medicines, 7(2), 125-137.

Piscitelli, S. C., Burstein, A. H., Chaitt, D., Alfaro, R. M., Falloon, J. (2000). Indinavir concentrations and St. John's wort. Lancet 255: 547-548.

Piscitelli, S. C., Burstein, A. H., Welden, N., Gallicano, K. D., Falloon, J. (2002). The effect of garlic supplements on the pharmacokinetics of saquinavir. Clin Infect Dis 34: 234-238.

Piscitelli, S. C., Formentini, E., Burstein A. H., Alfaro, R., Jagannatha, S., Falloon, J. (2002). Effect of milk thistle on the pharmacokinetics of indinavir in healthy volunteers.

Rajesh, R., Sudha, V., Varma, D., \& Sonika, S. (2012). Association between Medication Adherence Outcomes and Adverse Drug Reactions to Highly Active Antiretroviral Therapy in Indian Human Immunodeficiency VirusPositive Patients. Journal of young pharmacists : JYP, 4(4), 250-60.

Sandhu, R. S., Prescilla, R. P., Simonelli, T. M., Edwards, D. J. (2003). Influence of goldenseal root on the pharmacokinetics of indinavir. J Clin Pharmacol 2003.

Slain, D., Amsden, J. R., Khakoo, R. A., Fisher, M. A., Lalka, D., \& Hobbs, G. R. (2005). Effect of high-dose vitamin $\mathrm{C}$ on the steady-state pharmacokinetics of the protease inhibitor indinavir in healthy volunteers. Pharmacotherapy. 2005;25:165-70. doi: 10.1592/phco.25.2.165.56945. 
Tadesse, W., Mekonnen, A., Tesfaye, W., and Tadesse, Y. (2014). Self-reported adverse drug reactions and their influence on highly active antiretroviral therapy in HIV infected patients.

UNAIDS. (2008). HIV and AIDS estimates and data, 2007 and 2001. 2008 Report on the Global AIDS Epidemic.

UNAIDS. (2008). Joint United Nations Programme on HIV/AIDS (UNAIDS). Report on the global AIDS epidemic..

$\begin{array}{lllll}\text { UNAIDS. } & \text { (2010). } & \text { REPORT } & \text { ON } & \text { GLOBAL }\end{array}$ http://issuu.com/unaids/docs/unaids_globalreport_2010?mode $=$ window\&backgroundColor=\%23222222

25/01/2012, 09:45 HRS

UNAIDS. (2007). Message on the occasion of World AIDS Day 1 December. 2007.

Vickers, A., Jolly, B., \& Greenfield, M. (2006). Herbal medicine: women's views, knowledge and interaction with doctors: a qualitative study.

Visser, R., Ezzy, D., Bartos, M. (2000). Alternative or complementary? Nonallopathic therapies for HIV/AIDS. Altern Ther Health Med 2000;6:44-52.

WHO. (1978). World Health Organization Declaration of Alma-Ata: International Conference on Primary Health Care. Alma-Ata, USSR, 6-12 September 1978 http://www.who.int/publications/almaata_declaration_en.pdf. 3/10/2013, 11:00 hrs.

WHO. (2002). World Health Organization Traditional Medicine Strategy 2002-2005. Geneva, Switzerland.

WHO. (2005). Interim WHO clinical staging of HIV/AIDS and HIV/AIDS case definitions for surveillance. http://www.who.int/hiv/pub/guidelines/clinicalstaging.pdf assessed on 15/06/2012,5.30 pm

WHO. (2006). Antiretroviral therapy for HIV infection in adults and adolescents: Recommendations for a public health approach. $\underline{\text { http://www.who.int/hiv/pub/guidelines/artadultguidelines.pdf }}$

WHO. (2008). Traditional Medicine. Edited by centre M.

Wiwanitkit, V. (2003). The use of CAM by HIV-positive patients in Thailand. Complement Ther Med 2003;11:3941. 